\title{
Flow topology dynamics in a three-dimensional phase space for turbulent Rayleigh-Bénard convection
}

\author{
F.Dabbagh,${ }^{1,2, *}$ F.X.Trias, ${ }^{1, \dagger}$ A.Gorobets,,${ }^{3, \ddagger}$ and A.Oliva ${ }^{1, \S}$ \\ ${ }^{1}$ Heat and Mass Transfer Technological Center, Technical University of Catalonia, \\ ESEIAAT, c/Colom 11, 08222 Terrassa (Barcelona), Spain \\ ${ }^{2}$ Christian Doppler Laboratory for Multi-Scale Modeling of Multiphase Processes, \\ Johannes Kepler University, Altenbergerstraße 69, 4040 Linz, Austria \\ ${ }^{3}$ Keldysh Institute of Applied Mathematics, 4A, Miusskaya Sq., Moscow 125047, Russia
}

(Dated: February 4, 2020) 


\begin{abstract}
We study the flow topology dynamics in terms of the paramount non-linearities of enstrophy and strain production at hard turbulent regimes of Rayleigh-Bénard convection (RBC). To do so, a data-set of direct numerical simulations for an air turbulent RBC at Rayleigh numbers $R a=\left\{10^{8}, 10^{10}, 10^{11}\right\}$ is analysed. Considering the bulk dynamics therein, the classical $2 \mathrm{D}$ mean Lagrangian evolution of $Q_{\mathrm{G}}$ and $R_{\mathrm{G}}$ invariants of $\mathrm{G} \equiv \nabla \boldsymbol{u}$ is extended to $3 \mathrm{D}$ by decomposing $R_{\mathrm{G}}$ into two parts: the strain production, $R_{\mathrm{S}}$, and the enstrophy production, $\operatorname{tr}\left(\Omega^{2} \mathrm{~S}\right)$. In this way, the $3 \mathrm{D}$ phase space $\left(Q_{\mathrm{G}}, R_{\mathrm{S}}, \operatorname{tr}\left(\Omega^{2} \mathrm{~S}\right)\right)$ allows to identify separately the non-linear straining and rotational mechanisms in turbulence. The main resultant observations attest that, when the turbulent regime is notably hard, a rising local self-amplification of the velocity gradient takes place in strain-dominated areas. This process is strongly aided by vortex contraction. In concomitant, a pronounced increase in the linear contributions of vortex stretching is also identified, particularly relevant to strain-dominated slots.
\end{abstract}

\title{
I. INTRODUCTION
}

Many circulations in nature and industry, such as convection in the outer layer of the Sun, coherent structures in the Earth's atmosphere and oceans, mantle convection in the Earth's core, circulations in nuclear reactors and solar thermal power plants, are ruled by Rayleigh-Bénard convection (RBC). Namely, the turbulent dynamics therein is mainly stemmed from buoyancy variations in the dynamo of a thermally driven flow heated from below and cooled from above [1-3]. Besides the onset of flow structures, this dynamics becomes of significant complexity when the grade of turbulence and thermal forcing is very high, i.e. Rayleigh number $R a>10^{10}$. For instance, the rising self-sustained instabilities of $\mathrm{RBC}$ induce augmenting counter-gradient diffusion and energetic nonequilibriums between the buoyant production and viscous dissipation, which are mainly compensated by pressure fluctuations $[4,5]$. Although important features have been explored using direct numerical simulation (DNS) of RBC at hard turbulent regimes [6, 7], such as the stable boundary layers at $R a=2 \times 10^{12}$ [8] and the thermal plumes statistics at $R a=10^{12}$ [9]; many

\footnotetext{
* firas.dabbagh@jku.at

†xavi@cttc.upc.edu

† andrey.gorobets@gmail.com

$\S$ oliva@cttc.upc.edu
} 
questions regarding the coherent (small-scale) structures remain open. For instance, there is a lack of understanding about the flow topology and small-scale dynamics even though these components have a crucial contribution to the turbulent wind and energy budgets. They fully compose the main turbulence mechanisms, such as the evolution of hairpin vorticity dynamics, the non-Gaussianity, the strain/dissipation production and the cascade of kinetic energy [10-12]. In this regard, we aim to shed light on the following research question: how the physically meaningful non-linearities, such as the enstrophy generation (mechanism of vortex stretching) and the strain dissipation/production (mechanism of kinetic energy cascade), behave at hard turbulent regimes of $R B C$ ? The enstrophy and strain production terms constitute the key elements responsible for the self-amplifying nature of the velocity derivative $G \equiv \nabla \boldsymbol{u}$ in turbulence, which is believed to be a universal feature [10]. It is very remarkable the role of $G$ properties in investigating the structures of turbulent fluid motions [13] and the fine-scale dynamics [14, 15]. Another important relation in this regard is the so-called Tennekes and Lumely balance [11]: it states that for statistically stationary turbulence at high Reynolds numbers the viscous and production terms of enstrophy are in balance. Therefore, a detailed analysis of the small scale motions during their lifetime, in terms of these non-linearities, can provide fundamental perspectives about how the thermal turbulence is evolving with $R a$ in RBC. Moreover, apart from improving our understanding of buoyancy-driven turbulence, it can also provide highly valuable information needed to build up better turbulence models [16, 17].

In this paper we first outline a short description of the DNS configuration in Section II, together with an overview of previous findings regarding flow topology and small-scale dynamics for turbulent $\mathrm{RBC}$ at $R a=\left\{10^{8}, 10^{10}\right\}$, reported in [18]. In particular, we focus on showing the counterparts encountered at a new turbulence grade at $R a=10^{11}$. Afterwards, the total group of DNS data-set, i.e. $R a=\left\{10^{8}, 10^{10}, 10^{11}\right\}$, is used to explore the small-scale dynamics in terms of the enstrophy and strain production terms in Section III. Then, in order to have a more complete picture, the dynamics of small-scale kinetic-thermal interactions is studied in Section IV. Finally, all relevant results are summarised and conclusions are given in Section $\mathrm{V}$. 


\section{BACKGROUND}

In the above-explained context, well-known features of the small turbulent scales already encountered in different kinds of turbulent flows (e.g. isotropic turbulence [15], turbulent boundary layer [19], channel flow [20], turbulent mixing layer [21] and turbulent jets [22]),

were first explored in RBC by Dabbagh et al. [18] using DNS results at $R a=\left\{10^{8}, 10^{10}\right\}$. Namely, we simulated the dimensionless incompressible Navier-Stokes (NS) and thermal energy equations given by

$$
\begin{aligned}
\frac{D \boldsymbol{u}}{D t} & =\sqrt{\frac{P r}{R a}} \nabla^{2} \boldsymbol{u}-\nabla p+\boldsymbol{f}, \quad \nabla \cdot \boldsymbol{u}=0 \\
\frac{D T}{D t} & =\frac{1}{\sqrt{R a P r}} \nabla^{2} T
\end{aligned}
$$

where $D / D t=\partial / \partial t+(\boldsymbol{u} \cdot \nabla)$ is the Lagrangian derivative with time $t, p$ is the pressure, $T$ is the temperature, and $\boldsymbol{u}=(u, v, w)$ is the velocity field in Cartesian coordinates $\boldsymbol{x}=$ $(x, y, z)$. The governing equations (1) and (2) are written in non-dimensional form using the cavity height, $H$, the temperature difference between the upper and lower walls, $\Delta \Theta$, and the buoyant velocity, $U_{r e f}=\sqrt{\alpha g H \Delta \Theta}$ as references, where $\alpha$ is the volumetric thermal expansion coefficient and $g$ is the gravitational acceleration. The Boussinesq approximation is used to model the buoyancy forces, i.e. $\boldsymbol{f}=(0, T, 0)$. The studied configuration is shown in Figure 1a. Considering that the cavity is filled with air $(\operatorname{Pr}=\nu / \kappa=0.7)$, then the system depends only on the Rayleigh number, $R a=g \alpha \Delta \Theta H^{3} /(\nu \kappa)$, where $\nu$ is the kinematic viscosity and $\kappa$ is the thermal diffusivity. The global response is measured via the average Nusselt number, $N u$, given by

$$
N u=\sqrt{\operatorname{RaPr}}\langle v T\rangle_{A}-\frac{\partial\langle T\rangle_{A}}{\partial y},
$$

where the brackets operator $\langle\cdot\rangle$ represents the temporal average (likewise it denotes the ensemble average in the statistical analysis), and the subscript $A$ refers to the average over a horizontal $x-z$ plane at a given $y$-position. For details about the used numerical methods, algorithms, and verification of the DNS results, the reader is referred to our previous work [18]. In this work, we extend our DNS set with a new simulation at $R a=10^{11}$, computed on $8192 \mathrm{CPU}$ cores of the MareNostrum 4 supercomputer [23], and using the in-house STG code [24] on a grid of 5.7 billion nodes (see Figure 1b and Table I for details). Likewise the previous DNS [18], a fourth-order symmetry-preserving spatial discretization [25] has 
been used together with a self-adaptive second-order explicit time-integration scheme [26]. Finally, herein, the total data-set of DNS results at $R a=\left\{10^{8}, 10^{10}, 10^{11}\right\}$ is considered and analyzed, and a summary of parameters is given in Table I. The power-law scaling obtained, $N u \sim R a^{0.31}$, agrees very well with the fit of Grossmann-Lohse theory from Stevens et al. [27], and the DNS results of van der Poel et al. [9] for an air-filled cylindrical cell of aspect ratio $1 / 3$ and $R a$ up to $10^{12}$.

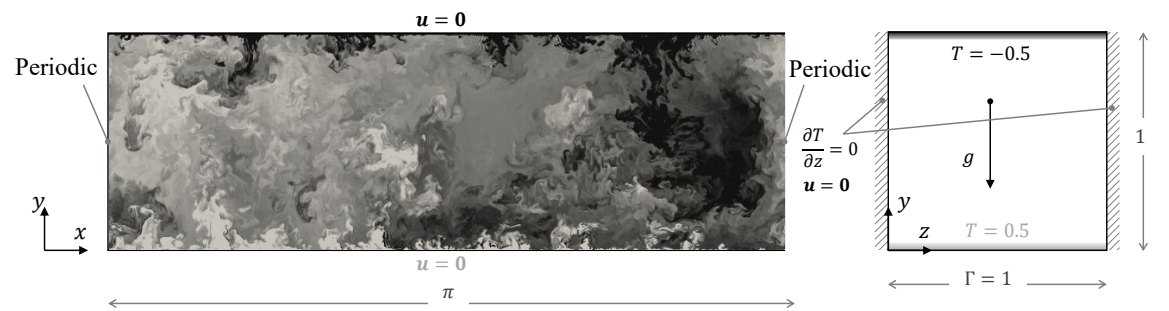

(a)

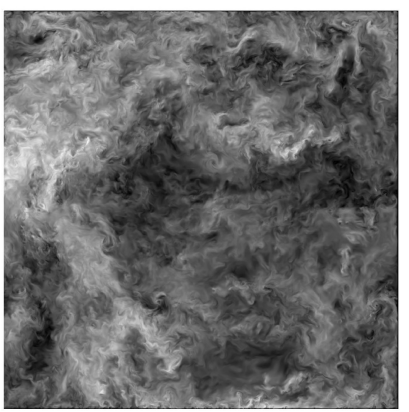

(b)

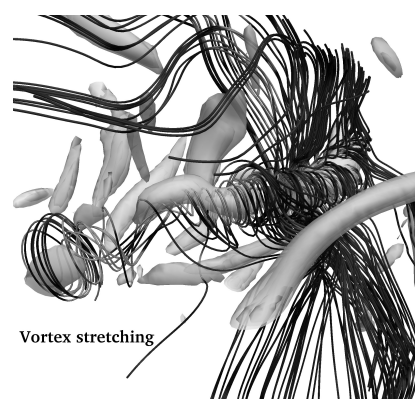

(c)

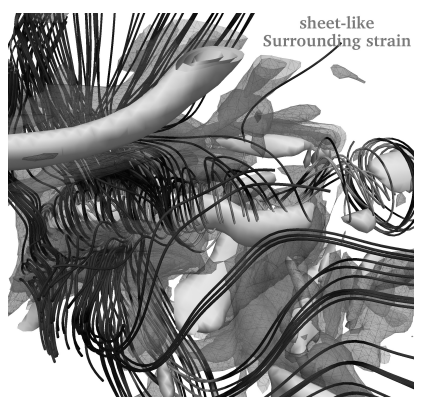

(d)

FIG. 1: (a) schema of the Rayleigh-Bénard configuration studied in Refs. [16, 18] and rendered with a developed instantaneous temperature field from the DNS at $R a=10^{10}$. (b) an instantaneous picture of $\|\boldsymbol{u}\|$ at $R a=10^{11}$ for a span-wise cross section. (c) structures of high positive $Q_{\mathrm{G}}$ iso-contours, which arrange like worms of intensive enstrophy. (d) displays the same picture of (c) in addition to visualization of the sheet-like strain structures that follow high negative values of $Q_{\mathrm{G}}$. Both (c) and (d) are taken from the DNS results at $R a=10^{8}$ and supplemented with some streamlines.

As mentioned above, results at $R a=\left\{10^{8}, 10^{10}\right\}$ allowed us to study relevant features of the small turbulent scales in RBC [18]. For example, we clearly observed the universal inclined "teardrop" shape of the joint PDF of the second, $Q_{\mathrm{G}}=-1 / 2 \operatorname{tr}\left(\mathrm{G}^{2}\right)$, and third invariant, $R_{\mathrm{G}}=-1 / 3 \operatorname{tr}\left(\mathrm{G}^{3}\right)$, of the velocity gradient tensor $\mathrm{G}$ in the $2 \mathrm{D}$ phase space $\left(Q_{\mathrm{G}}, R_{\mathrm{G}}\right)$ (see the similar tendency at $R a=10^{11}$ in Figure 2a). Notice that the flow is solenoidal, therefore, the first invariant is zero, i.e. $P_{\mathrm{G}}=\operatorname{tr}(\mathrm{G})=\nabla \cdot \boldsymbol{u}=0$. It is very common to use this phase space to classify the flow topology into four different classes [13, 15]. 
TABLE I: The physical and numerical parameters of the simulation adopted with refinement approaches to Grötzbach estimate, $\eta_{G r o ̈} \leq \pi \operatorname{Pr}^{1 / 2}((N u-1) R a)^{-1 / 4}[28] . \eta_{D N S}=(\Delta x \Delta y \Delta z)_{m a x}^{1 / 3}$ identifies the DNS grid size with $N_{B L}$ as the imposed number of nodes inside the thermal boundary layers (BLs) (exceeding Shishkina et al. [29] requirements). $\Delta t$ represents the time step in free-fall time units $[T U]$, and $\zeta$ indicates the temporal integration periods that guarantee a statistically stable turbulent heat transport. Quantitatively, $\zeta_{s t}$ lasts for several large eddy turnover times $\tau_{e d d y}$ during which, consecutive snapshots are gathered for the statistical analysis. Using the definition of $\tau_{\text {eddy }}=H / \boldsymbol{u}_{r m s}$, where $\boldsymbol{u}_{r m s}$ is the root mean square of bulk velocity, $\tau_{e d d y} \approx\{7,5,4\}[T U]$ for $R a=\left\{10^{8}, 10^{10}, 10^{11}\right\}$, respectively.

\begin{tabular}{|c|c|c|c|c|c|c|c|c|c|c|}
\hline$R a$ & $\eta_{G r \ddot{o}}$ & $\frac{(\Delta y=\Delta z)_{\max }}{\eta_{G r \ddot{m}}}$ & $\frac{\Delta x}{\Delta y_{\max }}$ & $\eta_{D N S}$ & $N_{x} \times N_{y} \times N_{z}$ & $N_{B L}$ & $\Delta t[T U]$ & $\zeta[T U$ & $\zeta_{s t}\left[\tau_{e d d y}\right]$ & $N u$ \\
\hline $10^{8}$ & 0. & 0.70 & 1.0 & $70 \times 10^{-3}$ & $08 \times 208$ & 9 & $45 \times 10^{-3}$ & 500 & 40 & 30.9 \\
\hline $10^{1 c}$ & .00246 & 0.92 & 1.36 & $2.50 \times 10^{-3}$ & $1024 \times 768 \times 768$ & 12 & $4.14 \times 10^{-4}$ & 200 & 10 & 128.1 \\
\hline $10^{1}$ & 0.00114 & 0.93 & 1.44 & $1.20 \times 10^{-3}$ & $2048 \times 1662 \times 1662$ & 13 & $1.66 \times 10^{-4}$ & 165 & 10 & 269.5 \\
\hline
\end{tabular}

Namely, $R_{\mathrm{G}}<0$ and $Q_{\mathrm{G}} \gg 0$ correspond to stable tube-like vortex-stretching structures, where positive enstrophy production is prevalent (see the instantaneous visualization in Figure 1c for the high positive $Q_{\mathrm{G}}$ iso-surfaces at $R a=10^{8}$, which are structured as the so-called worms [10], together with the streamlines). In the opposite quadrant, when $R_{\mathrm{G}}>0$ and $Q_{\mathrm{G}} \ll 0$, the straining production becomes dominant and it is mostly associated with strong unstable sheet-like viscous dissipative structures (the additional visualization shown in Figure 1d, that exposes the surrounding sheet-like strain, which is characterized by high negative values of $Q_{\mathrm{G}}$ ). The other two less frequent quadrants respectively correspond to ribbons of unstable vortex-compressing structures $\left(Q_{\mathrm{G}} \gg 0\right.$, and $\left.R_{\mathrm{G}}>0\right)$ and highly dissipative tube-like shape structures $\left(Q_{\mathrm{G}}<0\right.$ and $R_{\mathrm{G}}<0$, below the null-discriminant curve $\left.D_{\mathrm{G}}=(27 / 4) R_{\mathrm{G}}^{2}+Q_{\mathrm{G}}^{3}=0\right)$. In general, the joint PDF aspect rendered in Figure $2 \mathrm{a}$ indicates that most of the flow domain is occupied by large-scale (small mean gradients) dynamics around the origin, while the turbulent small scale motions obey substantially the tube-like vortex-stretching and the sheet-like viscous dissipation structures. The life-cycle of these scales, on the other hand, was interpreted by studying the mean evolution of $Q_{\mathrm{G}}$ and $R_{\mathrm{G}}$ invariants in the same $\left(Q_{\mathrm{G}}, R_{\mathrm{G}}\right)$ phase space. Meaning that, in the frame of a moving observer following a fluid particle, the local surrounding flow structures are essentially described by $\mathrm{G}$ and its invariants, i.e. $Q_{\mathrm{G}}$ and $R_{\mathrm{G}}$. Its change in time can be depicted by the Lagrangian dynamics of invariant quantities as proposed by Martín et al. [14] and Ooi et al. [15]. So, identical to that procedure, the mean trajectories of $\left(\left\langle D Q_{\mathrm{G}} / D t\right\rangle,\left\langle D R_{\mathrm{G}} / D t\right\rangle\right)$ were studied in RBC [18] and revealed the similar (universal?) characteristic clockwise spiraling behavior 
decaying towards the origin (see the new planer cycling trajectories plotted for $R a=10^{11}$ in Figure 2b). From Figure 2b, one can perceive how the local flow topology is altering, during its lifetime, from the viscous dissipation to the vortex-stretching and then the compressing structures, before vanishing in mean.

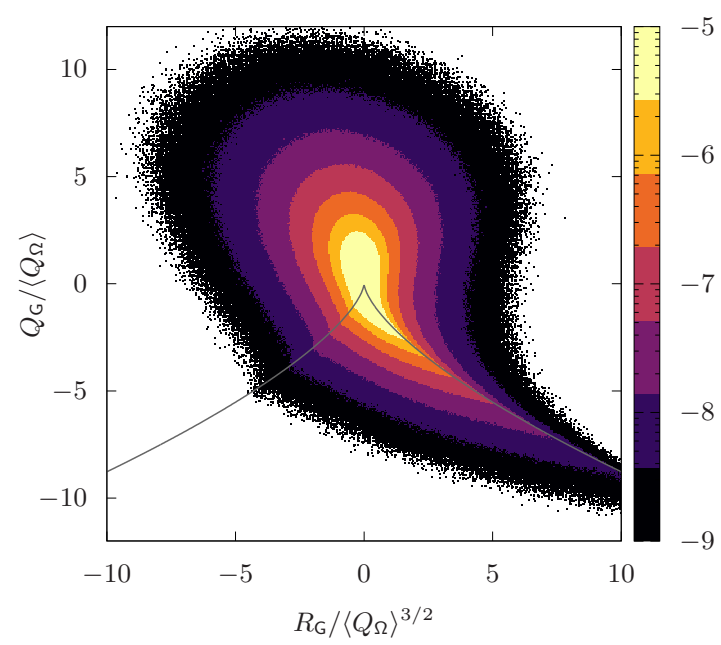

(a)

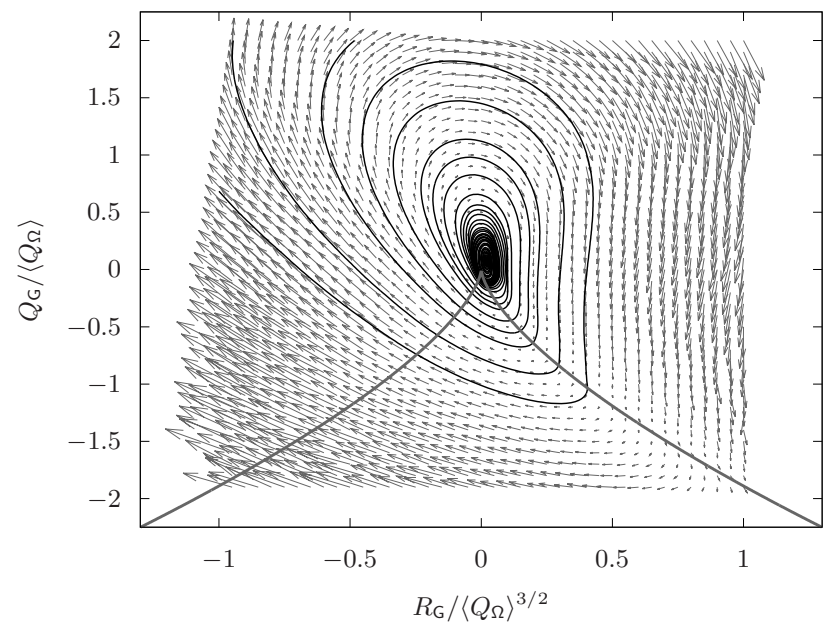

(b)

FIG. 2: (a) displays the joint PDF of normalized $Q_{\mathrm{G}}$ and $R_{\mathrm{G}}$ invariants in their own space, rendered on logarithmic scale, while (b) shows the conditional mean vectors of $\left\langle D Q_{\mathrm{G}} / D t\right\rangle$ and $\left\langle D R_{\mathrm{G}} / D t\right\rangle$ in the same plane together with the integral trajectories (black solid orbits). Both outcomes are taken from $R a=10^{11}$, through the bulk, where the solid continuous gray lines indicate the null-discriminant curve $D_{\mathrm{G}}=0$.

Apart from the above attributes, we also observed in [18] the preferential alignment of vorticity, $\boldsymbol{\omega}=\nabla \times \boldsymbol{u}$, with the intermediate eigenstrain vector $\boldsymbol{\lambda}_{2}$, and the vortexstretching vector, $\boldsymbol{\omega} S$, where $\mathbf{S}$ denotes the rate-of-strain tensor. This prevalent alignment was speculated as a universal feature of the geometrical structures in 3D turbulence [10]. By retrieving the similar aspects at $R a=10^{11}$, Figure 3 shows the PDF of $\cos \left(\boldsymbol{\omega}, \boldsymbol{\lambda}_{i}\right)=$ $\left(\boldsymbol{\omega} \cdot \boldsymbol{\lambda}_{i}\right) /\left(|\boldsymbol{\omega}|\left|\boldsymbol{\lambda}_{i}\right|\right)$ (Figure 3a) and $\cos (\boldsymbol{\omega}, \boldsymbol{\omega S})$ (Figure 3b), plotted together with the previous outcomes of turbulent RBC cases through the bulk. The new case, indicates similar trends and manifestation on the universal positiveness of the net enstrophy production, i.e. $\langle\boldsymbol{\omega}$. $\boldsymbol{\omega} S\rangle>0$ [18], which is revealed in Figure 3b. It is worth noticing that the new aspects, inspected in Figures 2 and 3 at $R a=10^{11}$, are very similar to $R a=10^{10}$ [18]. 


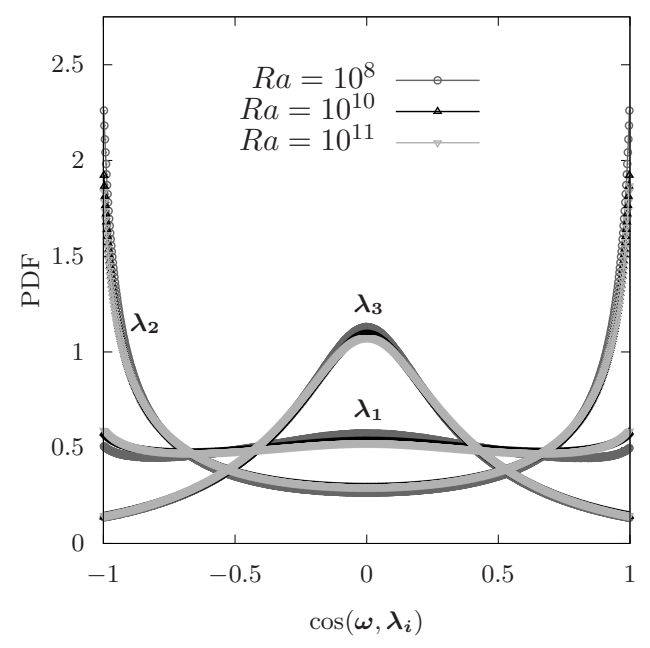

(a)

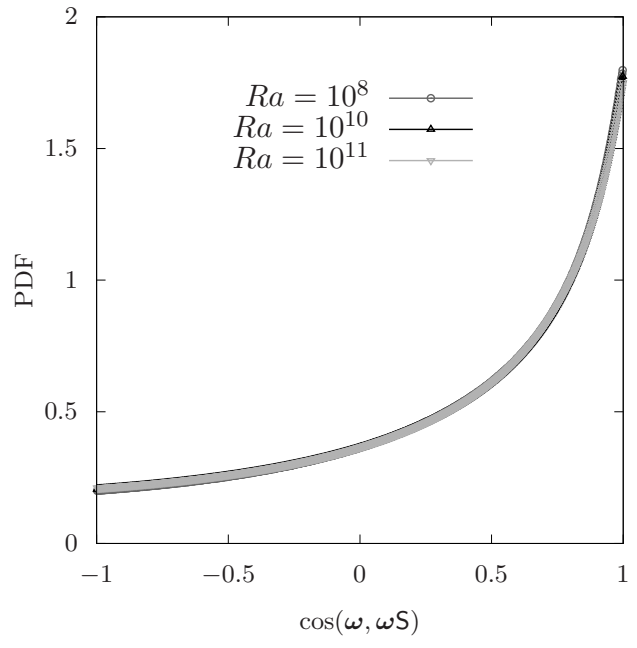

(b)

FIG. 3: PDF of vorticity alignments with the eigenvectors of the rate-of-strain tensor (a) and the vortex-stretching vector $\boldsymbol{\omega} \mathrm{S}$ (b), through the bulk, at $R a=\left\{10^{8}, 10^{10}, 10^{11}\right\}$.

\section{EVOLUTION IN A 3D PHASE SPACE}

Principally, in the current work, we seek to expose the role of strain generation, $R_{\mathrm{S}}=$ $-1 / 3 \operatorname{tr}\left(\mathrm{S}^{3}\right)$, and enstrophy production, $\operatorname{tr}\left(\Omega^{2} \mathrm{~S}\right)=R_{\mathrm{S}}-R_{\mathrm{G}}$, in controlling the evolution of small-scale dynamics. Here, $\mathrm{S}=1 / 2\left(\mathrm{G}+\mathrm{G}^{t}\right)$ and $\Omega=1 / 2\left(\mathrm{G}-\mathrm{G}^{t}\right)$ are the rate-of-strain and rate-of-rotation tensors, respectively. To do so, we expand our $2 \mathrm{D}\left(Q_{\mathrm{G}}, R_{\mathrm{G}}\right)$ evolution study [18] to the $3 \mathrm{D}$ phase space $\left(Q_{\mathrm{G}}, R_{\mathrm{S}}, R_{\mathrm{S}}-R_{\mathrm{G}}\right)$ by decomposing $R_{\mathrm{G}}=R_{\mathrm{S}}-\operatorname{tr}\left(\Omega^{2} \mathrm{~S}\right)$ into its two components, similar to Lüthi et al. [30]. In this way, we avoid the planner projection of trajectories onto $\left(Q_{\mathrm{G}}, R_{\mathrm{G}}\right)$, therewith the influence of increasing turbulence (non-linearity) is difficult to distinguish [18]. In other words, we better disclose the local self-amplification nature of $\mathrm{G}$ at high $R a$ in the evolution of flow topology by separating the production terms, i.e. $R_{\mathrm{S}}$ and $\operatorname{tr}\left(\Omega^{2} \mathrm{~S}\right)$, which were blent to $R_{\mathrm{G}}$ in the $\left(Q_{\mathrm{G}}, R_{\mathrm{G}}\right)$ space $[10,18]$. These third-moment non-linearities are essential parts of the enstrophy, $\boldsymbol{\omega}^{2}$, and total strain, $\mathrm{S}^{2}$, transport equations. Namely, by taking the gradient of the NS equations (1) and using the definition of the invariants $Q_{\Omega}=-1 / 2 \operatorname{tr}\left(\Omega^{2}\right)=1 / 4|\boldsymbol{\omega}|^{2}$ and $Q_{\mathrm{S}}=-1 / 2 \operatorname{tr}\left(\mathrm{S}^{2}\right)$, these transport equations read

$$
\begin{aligned}
& \frac{D Q_{\mathrm{S}}}{D t}=-3 R_{\mathrm{S}}+\operatorname{tr}\left(\Omega^{2} \mathrm{~S}\right)-\operatorname{tr}\left(\mathrm{SH}_{\mathrm{S}}\right) \\
& \frac{D Q_{\Omega}}{D t}=2 \operatorname{tr}\left(\Omega^{2} \mathrm{~S}\right)-\operatorname{tr}\left(\Omega \mathrm{H}_{\Omega}\right)
\end{aligned}
$$


where

$$
\begin{aligned}
& \mathrm{H}_{\mathrm{S}}=-\left(\mathrm{H}(p)-\frac{2 Q_{\mathrm{G}}}{3} \mathrm{I}\right)+\sqrt{\frac{P r}{R a}} \nabla^{2} \mathrm{~S}+1 / 2\left(\nabla \boldsymbol{f}+\nabla \boldsymbol{f}^{t}\right), \\
& \mathrm{H}_{\Omega}=\sqrt{\frac{P r}{R a}} \nabla^{2} \Omega+1 / 2\left(\nabla \boldsymbol{f}-\nabla \boldsymbol{f}^{t}\right),
\end{aligned}
$$

are second-order tensors that include the terms of the resultant pressure Hessian, $\mathrm{H}(p)$, the viscous diffusion terms of strain, $(\operatorname{Pr} / R a)^{1 / 2} \nabla^{2} \mathrm{~S}$, and rotation, $(\operatorname{Pr} / R a)^{1 / 2} \nabla^{2} \Omega$, and the gradient of the buoyancy forces, $\nabla \boldsymbol{f}$. On the other hand, it is worth to recall that this $3 \mathrm{D}$ phase space is a subset of the $5 \mathrm{D}$ phase space of invariants $\left(Q_{\mathrm{G}}, R_{\mathrm{G}}, Q_{\mathrm{S}}, R_{\mathrm{S}}, V^{2}\right)$ that fully describes the local flow topology for incompressible flows [14] and composes the cornerstone of turbulence models [31]. For the so-called "restricted Euler (RE) equations" (where the pressure, viscosity and buoyancy terms are neglected), the transport equations of these invariants read $[14,31]$

$$
\begin{aligned}
& \frac{D Q_{\mathrm{G}}}{D t}=-3 R_{\mathrm{G}} ; \quad \frac{D R_{\mathrm{G}}}{D t}=\frac{2}{3} Q_{\mathrm{G}}^{2} ; \\
& \frac{D Q_{\mathrm{S}}}{D t}=-2 R_{\mathrm{S}}-R_{\mathrm{G}} ; \quad \frac{D R_{\mathrm{S}}}{D t}=\frac{2}{3} Q_{\mathrm{G}} Q_{\mathrm{S}}+\frac{1}{4} V^{2} ; \quad \frac{D V^{2}}{D t}=-\frac{16}{3}\left(R_{\mathrm{S}}-R_{\mathrm{G}}\right) Q_{\mathrm{G}},
\end{aligned}
$$

where $V^{2}=4\left(\operatorname{tr}\left(\mathrm{S}^{2} \Omega^{2}\right)-2 Q_{\mathrm{S}} Q_{\Omega}\right)=|\mathbf{S} \boldsymbol{\omega}|^{2} \geq 0$ is the $L^{2}$-norm of vortex-stretching vector.

Identically to the 2D evolution procedure adopted in Refs. 15 and 18, the conditional 3D phase space $\left(Q_{\mathrm{G}}, R_{\mathrm{S}}, R_{\mathrm{S}}-R_{\mathrm{G}}\right)$ is analyzed using data from the bulk region $\left(V_{b u l k}=\{\boldsymbol{x}=\right.$ $(x, y, z) \mid 0.2 \leq y \leq 0.8 ; 0.2 \leq z \leq 0.8\})$ and dividing the following domain

$$
\left\{\begin{array}{ccc}
-2 \leq & Q_{\mathrm{G}} /\left\langle Q_{\Omega}\right\rangle & \leq 2 \\
-0.2 \leq \quad R_{\mathrm{S}} /\left\langle Q_{\Omega}\right\rangle^{3 / 2} & \leq 1.2 \\
-1 \leq & \left(R_{\mathrm{S}}-R_{\mathrm{G}}\right) /\left\langle Q_{\Omega}\right\rangle^{3 / 2} & \leq 1
\end{array}\right\}
$$

into $40 \times 40 \times 40$ bins. Therein, the average approach on the material derivative of $Q_{\mathrm{G}}, R_{\mathrm{S}}$ and $R_{\mathrm{S}}-R_{\mathrm{G}}$ is applied conditionally upon the invariants themselves. Namely, using a set of consecutive instantaneous flow fields collected during $\zeta_{s t}$ (see Table I), the following three components

$$
\begin{array}{r}
\left\langle\frac{D Q_{\mathrm{G}}}{D t} \mid-\frac{1}{2} \leq \frac{\left(Q_{\mathrm{G}}-Q_{\mathrm{G}}^{0}\right)}{\Delta Q_{\mathrm{G}}}<\frac{1}{2} ;-\frac{1}{2} \leq \frac{\left(R_{\mathrm{S}}-R_{\mathrm{S}}^{0}\right)}{\Delta R_{\mathrm{S}}}<\frac{1}{2} ;-\frac{1}{2} \leq \frac{\left(R_{\mathrm{S}}-R_{\mathrm{G}}\right)-\left(R_{\mathrm{S}}^{0}-R_{\mathrm{G}}^{0}\right)}{\Delta\left(R_{\mathrm{S}}-R_{\mathrm{G}}\right)}<\frac{1}{2}\right\rangle, \\
\left\langle\frac{D R_{\mathrm{S}}}{D t} \mid-\frac{1}{2} \leq \frac{\left(Q_{\mathrm{G}}-Q_{\mathrm{G}}^{0}\right)}{\Delta Q_{\mathrm{G}}}<\frac{1}{2} ;-\frac{1}{2} \leq \frac{\left(R_{\mathrm{S}}-R_{\mathrm{S}}^{0}\right)}{\Delta R_{\mathrm{S}}}<\frac{1}{2} ;-\frac{1}{2} \leq \frac{\left(R_{\mathrm{S}}-R_{\mathrm{G}}\right)-\left(R_{\mathrm{S}}^{0}-R_{\mathrm{G}}^{0}\right)}{\Delta\left(R_{\mathrm{S}}-R_{\mathrm{G}}\right)}<\frac{1}{2}\right\rangle, \\
\left\langle\frac{D\left(R_{\mathrm{S}}-R_{\mathrm{G}}\right)}{D t} \mid-\frac{1}{2} \leq \frac{\left(Q_{\mathrm{G}}-Q_{\mathrm{G}}^{0}\right)}{\Delta Q_{\mathrm{G}}}<\frac{1}{2} ;-\frac{1}{2} \leq \frac{\left(R_{\mathrm{S}}-R_{\mathrm{S}}^{0}\right)}{\Delta R_{\mathrm{S}}}<\frac{1}{2} ;-\frac{1}{2} \leq \frac{\left(R_{\mathrm{S}}-R_{\mathrm{G}}-\left(R_{\mathrm{S}}^{0}-R_{\mathrm{G}}^{0}\right)\right.}{\Delta\left(R_{\mathrm{S}}-R_{\mathrm{G}}\right)}<\frac{1}{2}\right\rangle,
\end{array}
$$


are computed to compose $3 \mathrm{D}$ vectors, where $\left(Q_{\mathrm{G}}^{0}, R_{\mathrm{S}}^{0}, R_{\mathrm{S}}^{0}-R_{\mathrm{G}}^{0}\right)$ are the center coordinates of the bin size $\left(\Delta Q_{\mathrm{G}}, \Delta R_{\mathrm{S}}, \Delta\left(R_{\mathrm{S}}-R_{\mathrm{G}}\right)\right)$. A robust converging of the rates therein, has been accomplished using a high number of samples $(>1000)$ per bin. The mean rate vectors are integrated along some paths to produce the conditional mean trajectories (CMTs) for the three turbulent cases $R a=\left\{10^{8}, 10^{10}, 10^{11}\right\}$ displayed in Figures 4a, 4b and 4c, respectively.

With a close inspection, one can deduce the following 3D scenario of dynamics in mean: at $R a=10^{8}$, the evolution of flow topology seems to be very weak in areas dominated by high positive values of strain production, $R_{\mathrm{S}}>0$. This consistently corresponds with the prevalence of the tube-like rotational geometry over strain slots in the bulk at that turbulent grade [18]. Therefore, the CMTs follow a planer $\left(Q_{\mathrm{G}}, R_{\mathrm{G}}\right)$ rotation near $R_{\mathrm{S}}=0$ moving on average from strain-dominated regions (Vieillefosse tail [32] at the null-discriminant plane $D_{\mathrm{G}}=0$ where $Q_{\mathrm{G}}<0$ and $R_{\mathrm{S}}$ dominates $\operatorname{tr}\left(\Omega^{2} \mathrm{~S}\right)$ ) towards the enstrophy-dominated areas, $Q_{\mathrm{G}}>0$, of vortex stretching, $R_{\mathrm{S}}-R_{\mathrm{G}}>0$, to regions of vortex contraction, $R_{\mathrm{S}}-R_{\mathrm{G}}<0$, and so on (see Figure 4a). They finally decay towards the origin $(0,0,0)$, vanishing the fluctuations in mean and leaving the large scales of the coherent uniform flow next to the origin [18].

By increasing the $R a$ number, the trajectories movement is drastically expanded to 3D helical tracks. They start the rotation in regions dominated by vortex stretching, $R_{\mathrm{S}}-R_{\mathrm{G}}>0$, and strain production, $R_{\mathrm{S}}>0$, preferentially located in strain-dominated areas, $-Q_{\mathrm{S}}$ or $Q_{\mathrm{G}}<0$ (see the facing $Q_{\mathrm{G}}-R_{\mathrm{S}}$ plane of combined CMTs for all cases, shown in Figure 4f). This helical movement becomes notable, stronger and further developed at $R a=\left\{10^{10}, 10^{11}\right\}$ (see Figures $4 \mathrm{~b}$ and $4 \mathrm{c}$, respectively). It manifests on: [C1] the enhanced local interaction $\boldsymbol{\omega} / \mathrm{S}$, which amplifies the linear contribution of vortex stretching in straindominated slots. Nearby observations suggest that, the axis of this helical behavior begins to be perpendicular to the intersection line of $Q_{\mathrm{G}}=0$ and $D_{\mathrm{G}}=0$ tent plane, in very high positive values of $R_{\mathrm{S}}-R_{\mathrm{G}}$ and $R_{\mathrm{S}}$ (see Figure $4 \mathrm{~d}$ and the facing $R_{\mathrm{S}}-\left(R_{\mathrm{S}}-R_{\mathrm{G}}\right)$ plane in Figure 4e). Afterwards, closer to the origin, the CMTs reverse their clockwise roll when they run downwards below, after crossing the $D_{\mathrm{G}}=0$ tent plane (see Figures $4 \mathrm{~d}$ and $4 \mathrm{f}$ ), and go back to their original rotation after flowing upwards, rotating towards the origin with an axis normal to $Q_{\mathrm{G}}-\left(R_{\mathrm{S}}-R_{\mathrm{G}}\right)$ plane near $R_{\mathrm{S}}=0$. This directional modification in 


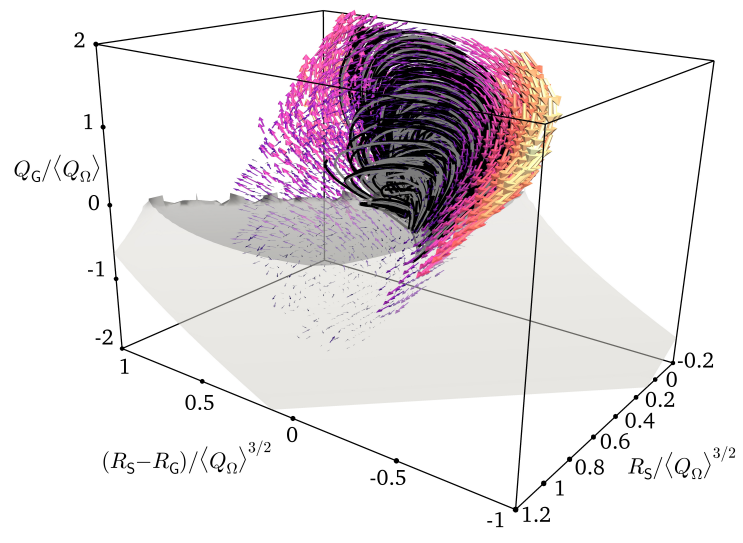

(a)

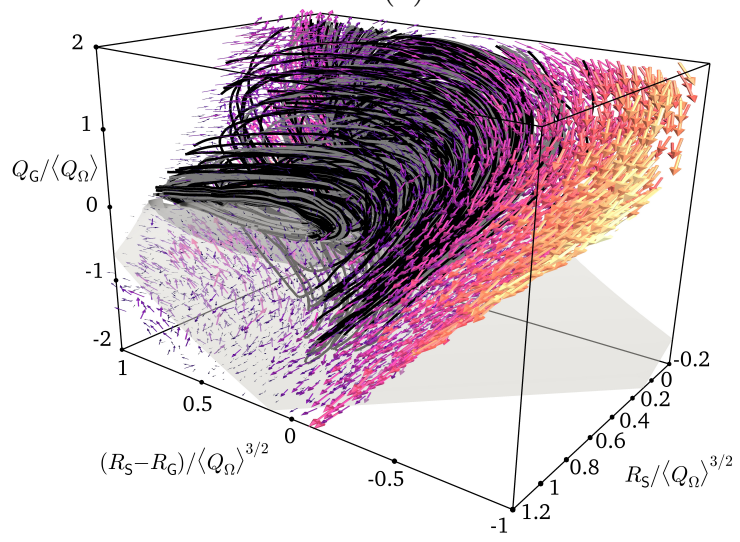

(c)

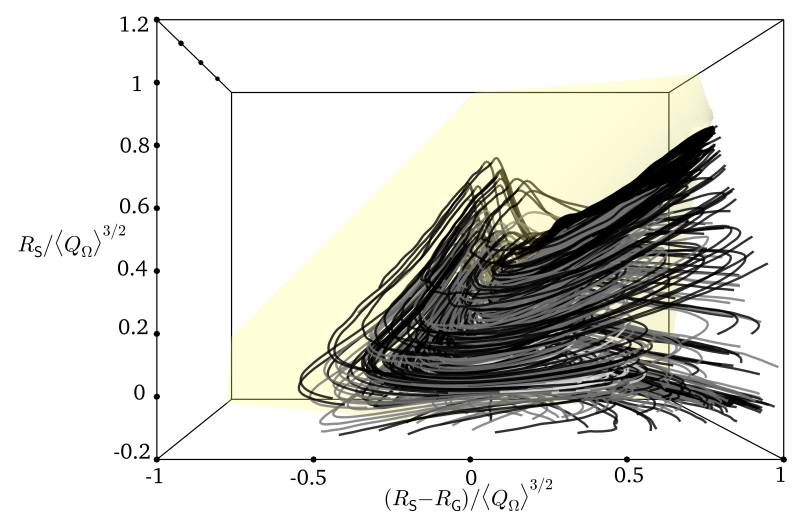

(e)

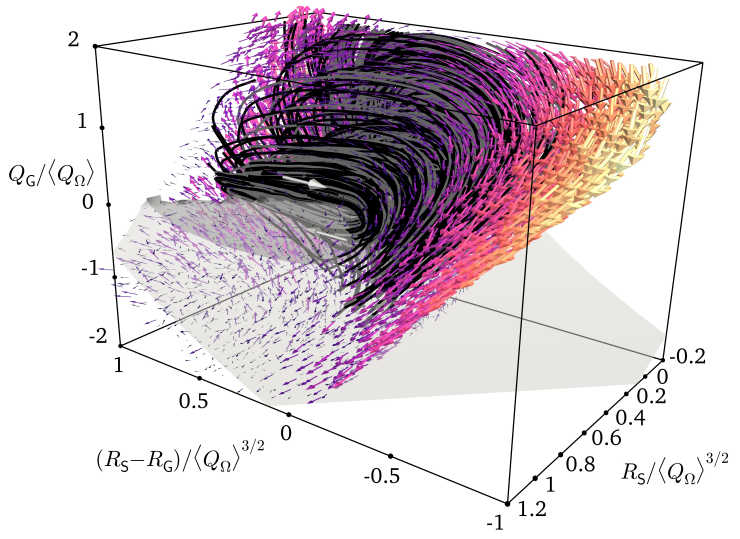

(b)

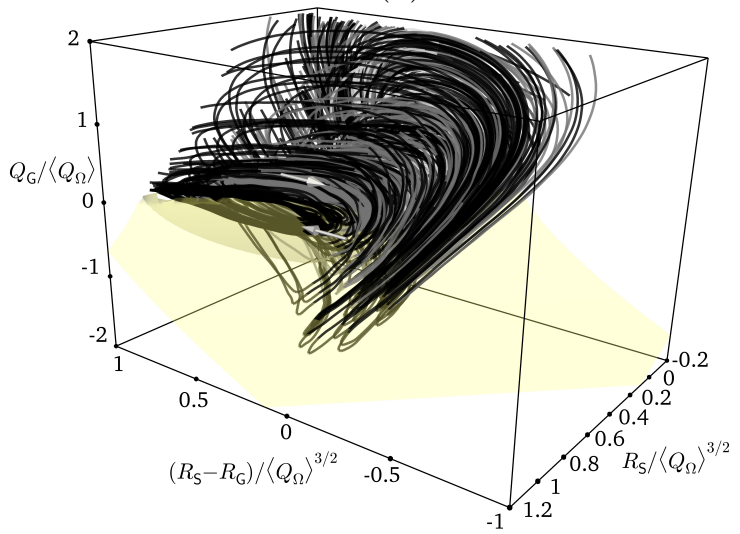

(d)

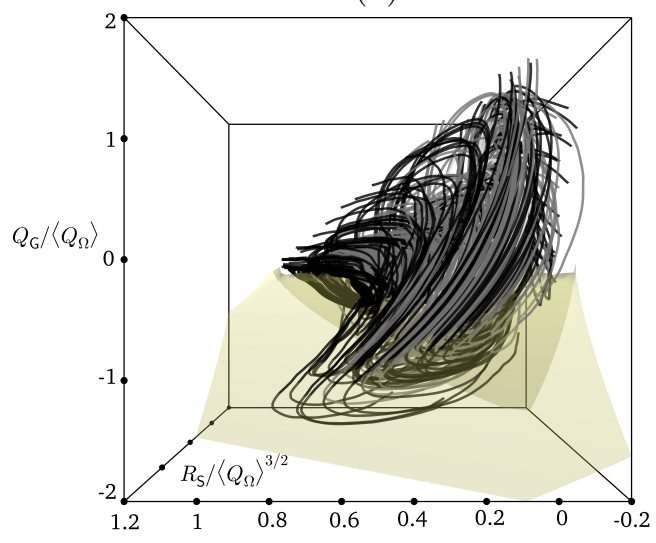

(f)

FIG. 4: (a), (b) and (c) represent the conditional mean vectors of $\left\langle D Q_{\mathrm{G}} / D t\right\rangle,\left\langle D R_{\mathrm{S}} / D t\right\rangle$ and $\left\langle D\left(R_{\mathrm{S}}-R_{\mathrm{G}}\right) / D t\right\rangle$ in

$\left(Q_{\mathrm{G}}, R_{\mathrm{S}}, R_{\mathrm{S}}-R_{\mathrm{G}}\right)$ space, colored and scaled proportionally to their magnitudes, and plotted together with their integral trajectories (black and gray lines) at $R a=10^{8}, 10^{10}$ and $10^{11}$, respectively, through $V_{\text {bulk }}$. (d) shows a combination of (a), (b) and (c) trajectories, plotted together by clear gray color for $R a=10^{8}$, dark gray color for $R a=10^{10}$ and black color for $R a=10^{11}$. (e) and (f) represent (d) in $R_{\mathrm{S}}-\left(R_{\mathrm{S}}-R_{\mathrm{G}}\right)$ and $Q_{\mathrm{G}}-R_{\mathrm{S}}$ facing views, respectively. The transparent tent-shape surface in all figures indicates the null-discriminant surface $D_{\mathrm{G}}=(27 / 4) R_{\mathrm{G}}^{2}+Q_{\mathrm{G}}^{2}=0$. 
the strain-dominated regions $-Q_{\mathrm{S}}$ is due to the opposite sign of $R_{\mathrm{S}}$ and $\operatorname{tr}\left(\Omega^{2} \mathrm{~S}\right)$ inside Eq. 4 .

While approaching the end, the vectors of mean dynamics obey a rapid downwards action in areas of enstrophy-dominated, $Q_{\mathrm{G}}>0$, vortex contraction, $R_{\mathrm{S}}-R_{\mathrm{G}}<0$, and strain production, $R_{\mathrm{S}}>0$. This action becomes stronger when increasing the turbulence grade, i.e. with higher $R a$ (see vectors in Figures $4 \mathrm{a}, 4 \mathrm{~b}$ and 4c, plotted by length and color range proportional to their magnitude). Relevant CMTs are found to travel extensively downwards in the strain-dominated areas $Q_{\mathrm{G}}<0$ towards high values of $R_{\mathrm{S}}>0$ as the $R a$ increases (see Figures 4a, 4b, 4c and 4f; for a better grasp of the evolution, see the movie of the CMTs for $R a=10^{10}$ [33]). This in turn confirms two additional issues: [C2] a local self-amplification of the turbulence background $\mathrm{G}$ and viscous straining, $-Q_{\mathrm{S}}$, in growing strain-dominated areas takes place at hard turbulent RBC and corresponds to amplified vorticity contracting events (note that positive values of $R_{\mathrm{S}}$ and negative ones of $\operatorname{tr}\left(\Omega^{2} \mathrm{~S}\right)$ strengthen $-Q_{\mathrm{S}}$ and vice versa in Eq. 4); [C3] a direct and local collaborative role of vortex compression to the dissipative actions and kinetic energy cascade. That is, vortex compression, $R_{\mathrm{S}}-R_{\mathrm{G}}<0$, aids the production of strain/dissipation, $R_{\mathrm{S}}>0$, and the kinetic energy cascade, on the contrary of the vortex stretching that suppresses the cascade [10].

In order to certify the conclusion $[\mathbf{C 1}]$, we investigate the local underlying physics of vortex-stretching vector, $\boldsymbol{\omega} S$. Namely, we propose to evaluate the mean value distribution of the squared magnitude of vortex-stretching vector, i.e. $V^{2}$, in condition on the joint PDF map of $\left(Q_{\mathrm{G}}, R_{\mathrm{S}}-R_{\mathrm{G}}\right)$ invariants. By doing so, one can determine whether the highest stretching is pertaining to the enstrophy-dominated $Q_{\Omega}$ regions or the strain-dominated $-Q_{\mathrm{S}}$ regions. Hence, the average values, $\left\langle V^{2} \mid\left(Q_{\mathrm{G}}, R_{\mathrm{S}}-R_{\mathrm{G}}\right)\right\rangle$, which are conditioned by the most probable joint PDF of $Q_{\mathrm{G}}$ and $\left(R_{\mathrm{S}}-R_{\mathrm{G}}\right)$ in their own space, are plotted and shown in Figure 5 a for $R a=10^{8}$. As expected, even at the lowest turbulent case $R a=10^{8}$, the maximum mean $V^{2}$ (black circle) is located in the positive enstrophy production $R_{\mathrm{S}}-R_{\mathrm{G}}>0$ and strain-dominated $Q_{\mathrm{G}}<0$ areas. Although the enstrophy production and $V^{2}$ remain large in the rotational dominated $Q_{\Omega}$ regions, its highest order of magnitude occur in the strain-dominated slots. Similar pictures are observed for the higher $R a$ numbers, identified by higher magnitudes for all elements. For a sharper comparison, the conditional averages of 
$V^{2}$ are determined (separated) in slots of $Q_{\Omega}$ and $-Q_{\mathrm{s}}$, as displayed in Figure 5b. Meaning that, we compute the mean value of $\left\langle V^{2} /\left\langle V^{2}\right\rangle\right\rangle$ in each bin $\Delta Q_{\Omega} /\left\langle Q_{\Omega}\right\rangle$ and $-\Delta Q_{\mathrm{s}} /\left\langle Q_{\Omega}\right\rangle$, which make up a total number of 300 bins. Again, for $R a=10^{8}$ (similar to the others), the stretching magnitude is much larger in strain-dominated areas than that in enstrophydominated ones; and the main contribution to the vortex stretching in these regions comes from effects associated with the local interaction $\omega / \mathrm{S}$. These findings, in particular the clear evidence in Figure 5b, are consistent with Tsinober et al. [34] where DNS results in a box with periodic boundary conditions at $R e_{\lambda}=75$ were used.

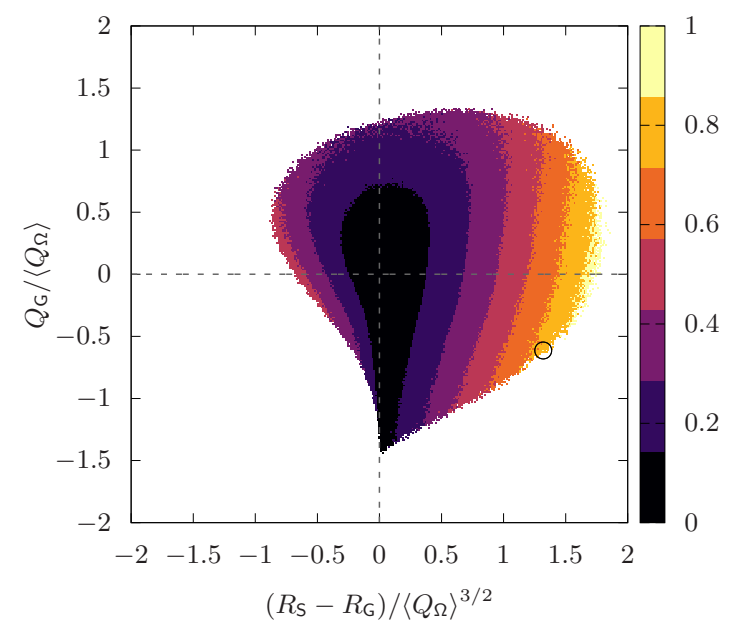

(a)

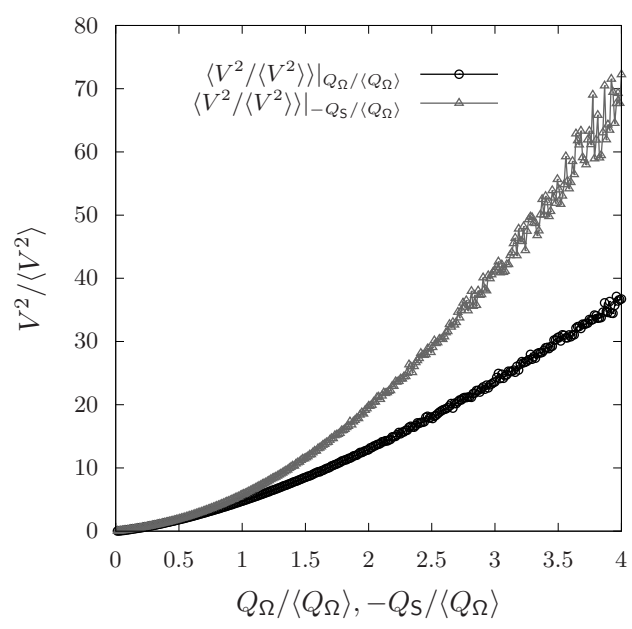

(b)

FIG. 5: (a) displays the average squared magnitude of vortex-stretching vector i.e. $\left\langle V^{2}\right\rangle$, conditioned on $\left(Q_{\mathrm{G}}, R_{\mathrm{S}}-R_{\mathrm{G}}\right)$ invariants space and normalized by its maximum. (b) shows the conditional averages of $V^{2}$ in slots of $Q_{\Omega}$ and $-Q_{\mathrm{S}}$. The data-set analyzed in both figures is obtained from $R a=10^{8}$ through the bulk, where the small black circle in (a) points out the maximum magnitude.

More investigations conducted for the same term $V^{2}$, but in condition of $Q_{\Omega}$ and $-Q_{\mathrm{S}}$ joint PDF map, are shown in Figure 6. This map provides physical information about the dominant flow topologies respect to the kinetic energy dissipation. For example, points of high enstrophy $Q_{\Omega}$ but very small dissipation $-Q_{\mathrm{S}}$ indicate solid-body rotational structures (vortex tubes), while points of strong dissipation but little enstrophy represent irrotational straining domination. Balanced distribution of $Q_{\Omega}=-Q_{\mathrm{S}}$ translates a vortex sheet topology, which normally occurs in the BLs. It was found in Dabbagh et al. [18] that the bulk geometries are mainly tube-like rotational with skewed map towards high $Q_{\Omega}$ at $R a=10^{8}$ 
(Figure 6a). However, at $R a=10^{10}$, the self-growing of $-Q_{\mathrm{s}}$ geometries was clearly identified, as demonstrated in Figure 6b. If we plot the average values of $\left\langle V^{2} \mid\left(Q_{\Omega},-Q_{\mathrm{S}}\right)\right\rangle$, similar to Figure $5 \mathrm{a}$, it can be observed that at $R a=10^{8}$ the maximum value (black circle) lies at $-Q_{\mathrm{S}} \sim 0.7$ in the dominated rotational topologies. This is natural since the vortex stretching obeys a dominant long-lived solid-body rotation. Note also the same maximum position of average $V^{2}$ at $Q_{\mathrm{G}} \sim-0.7$ in Figure $5 \mathrm{a}$. When the turbulence grade increases $\left(R a=10^{10}\right.$, which has the same trends as $R a=10^{11}$ ), the vortex tubes are breaking up by developing local (self-amplified) straining in the stretching contributions, and the maximum $\left\langle V^{2} \mid\left(Q_{\Omega},-Q_{\mathrm{s}}\right)\right\rangle$ shifts upwards in Figure $6 \mathrm{~b}$, towards higher values of $-Q_{\mathrm{s}}$. All these events confirm the conclusions $[\mathbf{C 1}]$ and $[\mathbf{C 2}]$.

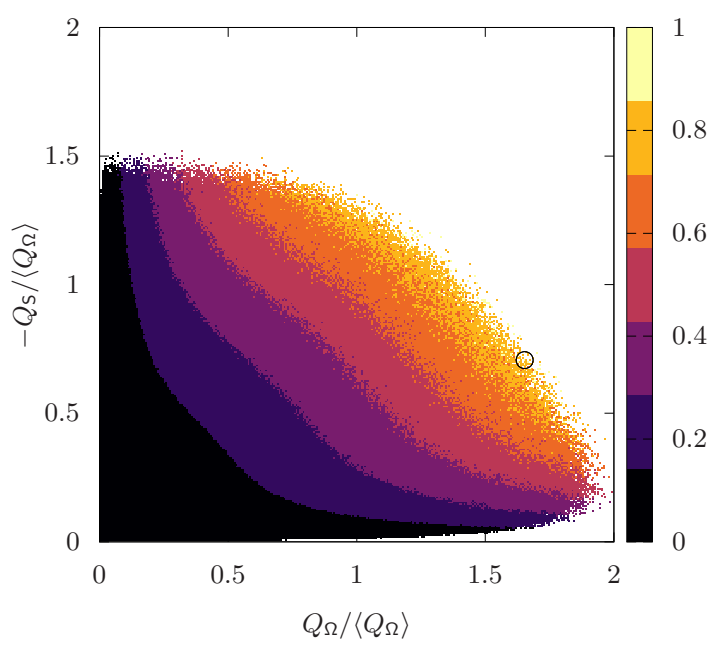

(a)

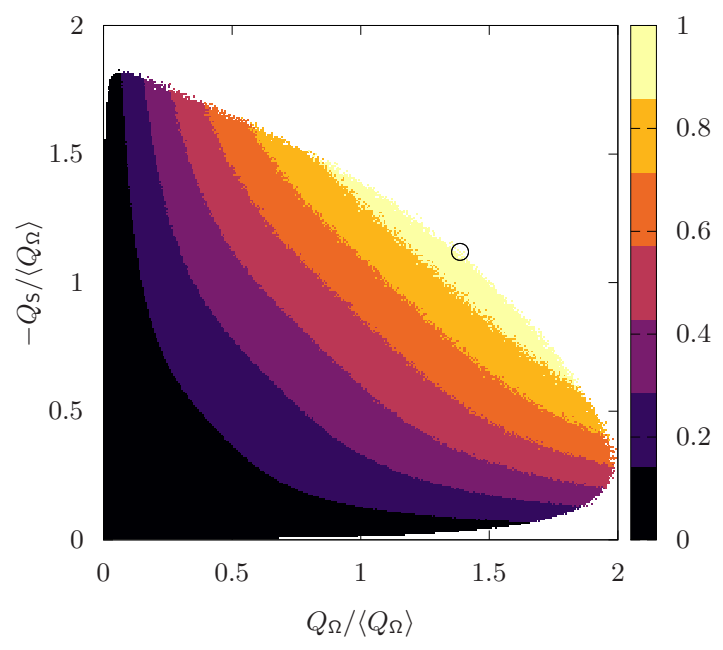

(b)

FIG. 6: The average squared magnitude of vortex-stretching vector, i.e. $\left\langle V^{2}\right\rangle$, conditioned on $\left(Q_{\Omega},-Q_{\mathrm{S}}\right)$ invariants space and normalized by its maximum. The statistics analyzed are taken from $R a=10^{8}$ in (a) and $R a=10^{10}$ in (b) through the bulk. Similar to Figure 5a, the small black circle indicates the maximum value of $\left\langle V^{2}\right\rangle$.

Our resultant evolution is consistent, to some extent, with the evolution dynamics studied by Lüthi et al. [30] for isotropic turbulence in the same 3D phase space. They found the same cyclical evolution in areas of positive enstrophy and strain productions, with the same rotation axis, leading to enstrophy dominated regions, $Q_{\mathrm{G}}>0$. It is noteworthy to evoke the statistical comparable aspects of isotropic turbulence with the bulk structures of RBC at $R a=10^{8}$, as showed in [18]. Therein, the joint PDF map of $\left(Q_{\Omega},-Q_{\mathrm{S}}\right)$, in $V_{b u l k}$, was found to be of similar distribution as the forced isotropic turbulence investigated in [15]. 
However, at higher $R a$-number, $R a=10^{10}$, the distribution deviates (Figure 6b). Hence, such a discrepancy respect to the results obtained in [30] for isotropic turbulence may be produced by our higher turbulence grades, the confinement effect of our RBC and the different mechanism of injecting kinetic energy into the system, i.e. by the dissipation of thermal plumes.

For a rough comparison, we have simulated the evolution of the $5 \mathrm{D}$ phase space of invariants $\left(Q_{\mathrm{G}}, R_{\mathrm{G}}, Q_{\mathrm{S}}, R_{\mathrm{S}}, V^{2}\right)$ for the $\mathrm{RE}$ equations projected onto our 3D phase space $\left(Q_{\mathrm{G}}, R_{\mathrm{S}}, R_{\mathrm{S}}-R_{\mathrm{G}}\right)$. To do so, we have integrated Eqs. 8 and 9 explicitly from multiple initial values for $\left(Q_{\mathrm{G}}, R_{\mathrm{G}}, Q_{\mathrm{S}}, R_{\mathrm{S}}, V^{2}\right)$, and their evolution have been projected and plotted in the 3D phase space $\left(Q_{\mathrm{G}}, R_{\mathrm{S}}, R_{\mathrm{S}}-R_{\mathrm{G}}\right)$ in Figure 7 a together with the tent plane $D_{\mathrm{G}}=0$. One can note how the trajectories start from small values of $R_{\mathrm{S}}-R_{\mathrm{G}}>0$ and $R_{\mathrm{S}}>0$ in the strain-dominated regions, $Q_{\mathrm{G}}<0$, asymptotic to the left-side of the $D_{\mathrm{G}}=0$ wire-mesh. Then, they expand and rotate in the quadrant of positive enstrophy and strain productions, $R_{\mathrm{S}}-R_{\mathrm{G}}>0$ and $R_{\mathrm{S}}>0$, towards high values in the rotation-dominated areas, $Q_{\mathrm{G}}>0$ (see Figures $7 \mathrm{a}$ and $7 \mathrm{~b}$ ). This behavior is similar to the dynamics observed in the DNS (see Figure 4e, where the rotation axis is similar to Figure 7b), but the spiraling direction is reversed and is preferentially located in strain-dominated areas, $Q_{\mathrm{G}}<0$ (see Figure 4f). It can be deduced that at high turbulence grade, the effect of the non-linear advection (convective term) appears in both cases and concentrates, for the RE model, in the centered vorticity tube-like filaments/worms, where the vortex stretching and intensive enstrophy are dominant, i.e. $R_{\mathrm{S}}-R_{\mathrm{G}}>0, Q_{\mathrm{G}}>0$ and $R_{\mathrm{S}}>0$. In particular, in these areas where $Q_{\Omega}$ is dominant, for the DNS the enstrophy production is in an approximate balance with the viscous destruction of rotation $-\operatorname{tr}\left(\Omega(\operatorname{Pr} / R a)^{1 / 2} \nabla^{2} \Omega\right)$, as discussed by Tsinober [10]. Hence, the influence of the natural turbulence remains predominantly in the strain-dominated regions of the vortex-stretching patterns $[\mathbf{C 1}]$, and is primarily returned to the pressure Hessian term. This conforms well with Tsinober et al. [34] who found that in regions of high strain the pressure Hessian even enhances the growth of stretching. On the other hand, Lüthi et al. [30], in their similar 3D phase space for isotropic turbulence, and other detailed studies [35, 36], have corroborated that the RE dynamics are essentially counteracted by the deviatoric non-local part of the pressure Hessian and not by the viscous term. Afterwards, the RE trajectories continue asymptotic to the right-side of $D_{\mathrm{G}}=0$ wire- 
mesh, towards negative values of $R_{\mathrm{S}}<0$, since negative enstrophy production, $\operatorname{tr}\left(\Omega^{2} \mathrm{~S}\right)<0$, leads to negative values of $Q_{\mathrm{G}}$ in its evolution equation (first part of Eq. 8) with $R_{\mathrm{S}}<0$. In the DNS, all CMTs are twisted towards $R_{\mathrm{S}}>0$ to be converged to the origin in a cyclic evolution.

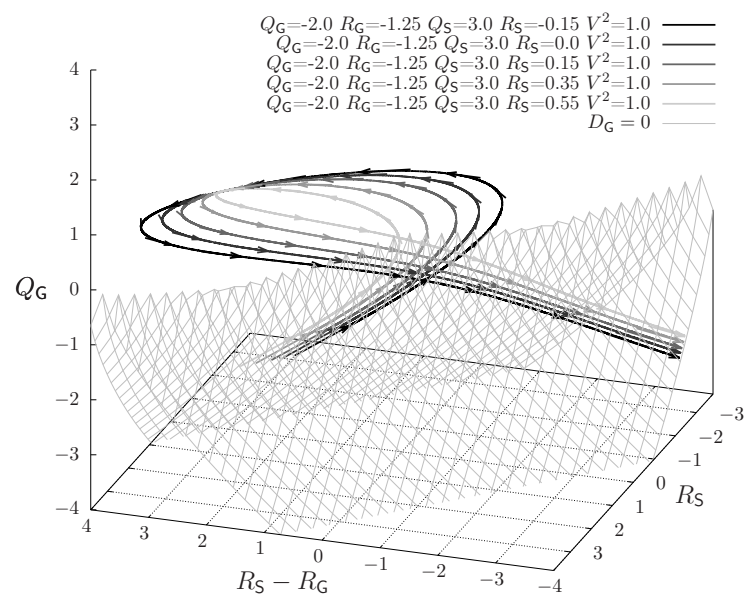

(a)

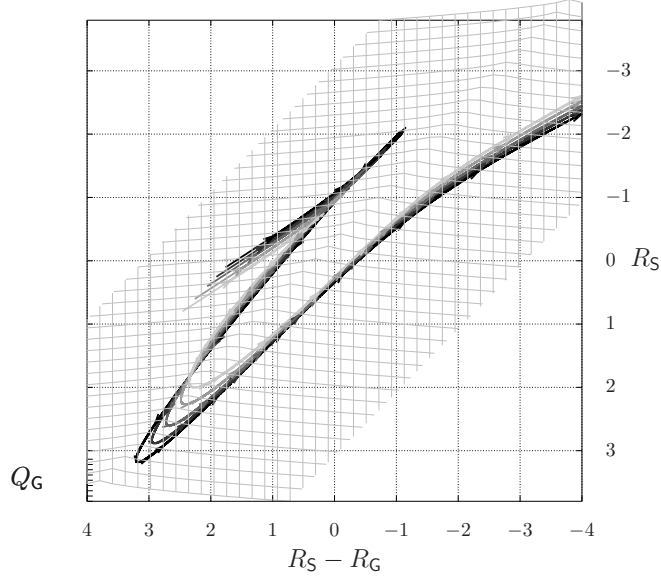

(b)

FIG. 7: Evolution trajectories of the 5D phase space invariants $\left(Q_{\mathrm{G}}, R_{\mathrm{G}}, Q_{\mathrm{S}}, R_{\mathrm{S}}, V^{2}\right)$, given in Eqs. 8,9 for the restricted Euler model, and plotted together with their vectors in the $3 \mathrm{D}$ phase space $\left(Q_{\mathrm{G}}, R_{\mathrm{S}}, R_{\mathrm{S}}-R_{\mathrm{G}}\right)$. One track (solution) is generated for each set of initial values for the $5 \mathrm{D}$ phase space of invariants. The null-discriminant $D_{\mathrm{G}}=0$ wire-mesh plane is drawn therewith. The trajectories are displayed in two views, from the side in (a) and from the top in (b).

\section{EVOLUTION IN A KINETIC-THERMAL 2D PHASE SPACE}

To study the turbulent heat flux and buoyant production mechanisms in turbulent RBC, the small-scale dynamics relevant to thermals was also reported in [18], for $R a=\left\{10^{8}, 10^{10}\right\}$. Namely, we applied the same approach as in the $2 \mathrm{D}$ phase space $\left(Q_{\mathrm{G}}, R_{\mathrm{G}}\right)$ but using the invariants of the traceless part of velocity-times-temperature gradient tensor, i.e. $\left(Q_{\tilde{\mathrm{G}}_{\theta}}, R_{\tilde{\mathrm{G}}_{\theta}}\right)$, where $\tilde{\mathrm{G}}_{\theta}=\mathrm{G}_{\theta}-1 / 3 \operatorname{tr}\left(\mathrm{G}_{\theta}\right) \mathrm{I}$, with $\mathrm{G}_{\theta}=\nabla(\boldsymbol{u} T)$. The newly proposed invariants demonstrated a direct picture on the small-scale kinetic-thermal interaction dynamics and thermal plumes evolution in RBC. For example, the statistical analysis of the joint $\operatorname{PDF}$ map $\left(Q_{\tilde{\mathrm{G}}_{\theta}}, R_{\tilde{\mathrm{G}}_{\theta}}\right)$, through the total domain, held a symmetric aspect respect to $R_{\tilde{\mathrm{G}}_{\theta}}=0$ (almost similar to a Gaussian flow field) that directly follows the universal log-normal statistics distribution of the thermal plumes geometries. It was noted that the CMTs of $\left(\left\langle D Q_{\tilde{\mathrm{G}}_{\theta}} / D t\right\rangle,\left\langle D R_{\tilde{\mathrm{G}}_{\theta}} / D t\right\rangle\right)$ 
travel downwards to expose the sheet-like plumes dynamics (roots) within the BLs, and upwards to exhibit the evolution of mushroom-like plumes that expands in the bulk. At $R a=10^{8}$, the trajectories showed two skew-symmetric converging origins, which indicated the contribution of the hot and cold mushroom-like plumes, into the large coherent scales of heat flux. However, at $R a=10^{10}$ the CMTs were traveling upwards in shorter tracks to demonstrate the reduced lifetime of mushroom-like plumes under the influence of the selfamplified dissipation and hard mixing. In consequence, all trajectories showed a zero-value converging origin in that turbulent case. In this work, we have extended the range up to $R a=10^{11}$ (results are shown in Figure 8). Similar to $R a=10^{10}$, we have noticed the zerovalue converging origin of CMTs plotted across the whole domain (Figure 8a), and a similar joint $\operatorname{PDF}\left(Q_{\tilde{\mathrm{G}}_{\theta}}, R_{\tilde{\mathrm{G}}_{\theta}}\right)$ distribution with increasing events (points) located below $D_{\tilde{\mathrm{G}}_{\theta}}=0$ (Figure 8b). This confirms our previous findings: the mushroom-like thermal plumes are abundantly emitted in the BLs and scattered in the bulk under the impact of self-amplified turbulence background. Hence, they do not contribute to the mean (large scale) heat flux, as well, at $R a=10^{11}$. However, a very interesting feature can be drawn; namely, the evolution of sheet-like plumes vanishes at the expense of increasing the evolution of mushroom-like plumes at $R a=10^{11}$. This has been deduced from the total up-warded tracks of CMTs below the null-discriminant curve $D_{\tilde{\mathrm{G}}_{\theta}}=0$ at $Q_{\tilde{\mathrm{G}}_{\theta}}<0$ (see Figure $8 \mathrm{~b}$ ). They arise as a true reflection of the increased emitting mushroom-like plumes in the BLs and the placements of more events below $D_{\tilde{\mathrm{G}}_{\theta}}=0$ in Figure 8a. It was found in Ref. 18, that $D_{\tilde{\mathrm{G}}_{\theta}}=0$ curve can separate the sheet-like plumes (roots) evolution (which move downwards at $R a=\left\{10^{8}, 10^{10}\right\}$ below $D_{\tilde{\mathrm{G}}_{\theta}}=0$ ) in the BLs, from the arisen mushroom-like plumes expanded into the bulk (which move upwards above $D_{\tilde{\mathrm{G}}_{\theta}}=0$ and converge to the origin). Accordingly, this event supports the fact that the horizontal waves, which travel in the BLs and interact with each other to compose the sheet-like plumes [37], become weaker at $R a=10^{11}$. In other words, less stabilizing effects are pronounced in the BLs, which become even thinner at $R a=10^{11}$, and tend to lose its laminar status.

\section{SUMMARY AND CONCLUSIONS}

On the base of a fully resolved DNS data-set for an air buoyancy-driven RBC, the behaviour of the small scale dynamics inside the bulk region has been explored at different 


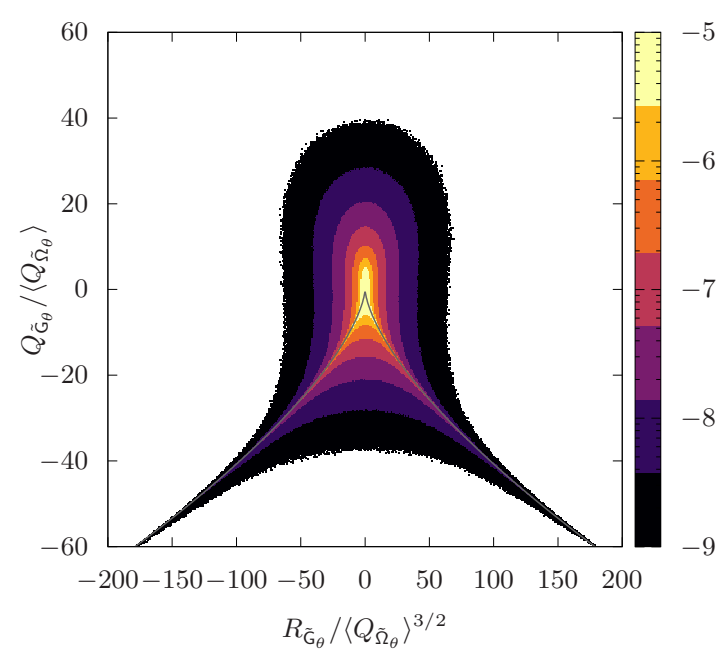

(a)

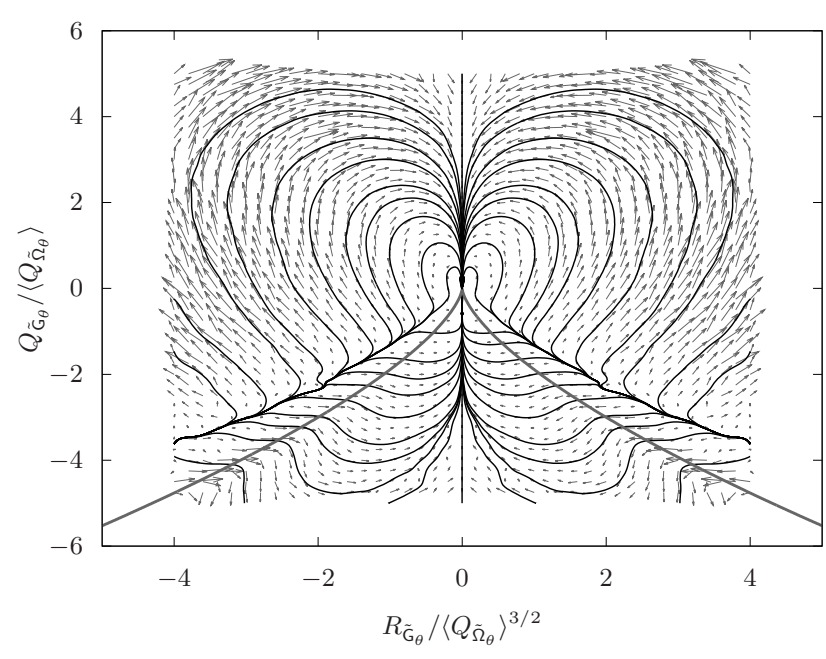

(b)

FIG. 8: The same representation as in Fig. 2 considering (a) the joint PDF of $\left(Q_{\tilde{\mathrm{G}}_{\theta}}, R_{\tilde{\mathrm{G}}_{\theta}}\right)$ and $(\mathrm{b})\left\langle D Q_{\tilde{\mathrm{G}}_{\theta}} / D t\right\rangle,\left\langle D R_{\tilde{\mathrm{G}}_{\theta}} / D t\right\rangle$ in the $\left(Q_{\tilde{\mathrm{G}}_{\theta}}, R_{\tilde{\mathrm{G}}_{\theta}}\right)$ space. The results correspond to the RBC simulation at $R a=10^{11}$ through the total domain. The gray solid line in (a) and (b) represents $D_{\tilde{\mathrm{G}}_{\theta}}=0$.

turbulent regimes, $R a=\left\{10^{8}, 10^{10}, 10^{11}\right\}$. By adopting a similar approach as Lüthi et al. [30], we have expanded the 2D Lagrangian evolution of $Q_{\mathrm{G}}$ and $R_{\mathrm{G}}$ invariants, previously reported in Dabbagh et al. [18], to a 3D phase space by decomposing $R_{\mathrm{G}}$ into two parts: the strain production, $R_{\mathrm{S}}$, and the enstrophy production, $\operatorname{tr}\left(\Omega^{2} \mathrm{~S}\right)$. By doing so, it has been demonstrated the existence of a 3D cyclical evolution of G state, strongly expanded at the hard turbulent cases, i.e. $R a=\left\{10^{10}, 10^{11}\right\}$. This cyclical rotation starts preferentially in the strain-dominated areas from regions of high positive magnitudes of vortex stretching and strain production. Following its life-cycle, the concluded outcomes manifest a rising self-amplification of $\mathrm{G}$ in the strain-dominated area, as the turbulence grade increases. This process is accompanied by a strong enhanced vortex contraction and amplified linear contributions of vortex stretching, which are particularly related to the strain-dominated slots. Regarding the evolution of the small scale thermals, the $2 \mathrm{D}$ evolution of $Q_{\tilde{\mathrm{G}}_{\theta}}$ and $R_{\tilde{\mathrm{G}}_{\theta}}$, invariants of $\tilde{\mathrm{G}}_{\theta}=\mathrm{G}_{\theta}-1 / 3 \operatorname{tr}\left(\mathrm{G}_{\theta}\right) \mathrm{I}$, with $\mathrm{G}_{\theta}=\nabla(\boldsymbol{u} T)$, was investigated for first time in Dabbagh et al. [18], at $R a=\left\{10^{8}, 10^{10}\right\}$, showing a direct description of thermal plumes evolution in RBC. Here, it has been extended by considering the case at $R a=10^{11}$. At this turbulent regime, we have noticed a clear tendency of reducing the evolution of sheet-like plumes in the BLs at the expense of the increasing emission of mushroom-like plumes. This may support the fact that a reduced laminarization of the BL occurs at $R a=10^{11}$. 
It would be of great interest to extend this analysis to even higher $R a$-numbers; especially up to the point where BLs reach a turbulent regime. However, nowadays DNS of buoyancy-driven flows is still limited to relatively low $R a$-numbers because the convective term produces far too many relevant scales of motion. Therefore, the development of better subgrid heat flux models in large-eddy simulation is part of our near future research plans.

\section{ACKNOWLEDGMENTS}

F.X.T., F.D. and A.O. are financially supported by the Ministerio de Economía y Competitividad, Spain (ENE2017-88697-R). F.X.T. is supported by a Ramón y Cajal postdoctoral contract (RYC-2012-11996). F.D. is supported by the Austrian Federal Ministry for Digital and Economic Affairs, the National Foundation for Research, Technology and Development, and the K1MET center for metallurgical research in Austria (www.k1-met.com). Calculations have been performed on the IBM MareNostrum 4 supercomputer at the BSC (PRACE 15th Call, Ref. 2016163972, "Exploring new frontiers in Rayleigh-Bénard convection"). Preliminary simulations were carried out using computational resources of MCC NRC "Kurchatov Institute", http://computing.nrcki.ru/. The authors thankfully acknowledge these institutions.

[1] F. Chillà and J. Schumacher, New perspectives in turbulent Rayleigh-Bénard convection, The European Physics Journal E 35, 58 (2012).

[2] G. Ahlers, S. Grossmann, and D. Lohse, Heat transfer and large scale dynamics in turbulent Rayleigh-Bénard convection, Reviews of Modern Physics 81, 503 (2009).

[3] E. D. Sigga, High Rayleigh number convection, Annual Reviews of Fluid Mechanics 26, 137 (1994).

[4] R. Togni, A. Cimarelli, and E. D. Angelis, Physical and scale-by-scale analysis of RayleighBénard convection, Journal of Fluid Mechanics 782, 380 (2015).

[5] K. Hanjalić, One-point closure models for buoyancy-driven turbulent flows, Annual Reviews of Fluid Mechanics 34, 321 (2002). 
[6] G. Amati, K. Koal, F. Massaioli, K. R. Sreenivasan, and R. Verzicco, Turbulent thermal convection at high Rayleigh numbers for a boussinesq fluid of constant Prandtl number, Physics of Fluids 17, 121701 (2005).

[7] R. A. J. M. Stevens, R. Verzicco, and D. Lohse, Radial boundary layer structure and Nusselt number in Rayleigh-Bénard convection, Journal of Fluid Mechanics 643, 495 (2010).

[8] R. J. A. M. Stevens, D. Lohse, and R. Verzicco, Prandtl and Rayleigh number dependence of heat transport in high Rayleigh number thermal convection, Journal of Fluid Mechanics 688, $31(2011)$.

[9] E. P. van der Poel, R. Verzicco, S. Grossmann, and D. Lohse., Plume emission statistics in turbulent Rayleigh- Bénard convection, Journal of Fluid Mechanics 772, 5 (2015).

[10] A. Tsinober, An Informal Introduction to Turbulence, Vol. 63 (Fluid Mechanics and its Applications, Kluwer Academic Publishers, 2001).

[11] H. Tennekes and J. L. Lumley, A First Course in Turbulence (MIT Press, 1972).

[12] K. R. Sreenivasan and R. A. Antonia, The phenomenology of small-scale turbulence, Annual Review of Fluid Mechanics 29, 435 (1997).

[13] M. S. Chong, A. E. Perry, and B. J. Cantwell, A general classification of three-dimensional flow fields, Physics of Fluids A 2, 765 (1990).

[14] J. Martín, A. Ooi, M. S. Chong, and J. Soria, Dynamics of the velocity gradient tensor invariants in isotropic turbulence, Physics of Fluids 10, 2336 (1998).

[15] A. Ooi, J. Martin, J. Soria, and M. S. Chong, A study of the evolution and characteristics of the invariants of the velocity-gradient tensor in isotropic turbulence, Journal of Fluid Mechanics 381, 141 (1999).

[16] F. Dabbagh, F. X. Trias, A. Gorobets, and A. Oliva, A priori study of subgrid-scale features in turbulent Rayleigh-Bénard convection, Physics of Fluids 29, 105103 (2017).

[17] R. Togni, A. Cimarelli, and E. D. Angelis, Resolved and subgrid dynamics of RayleighBénard convection, Journal of Fluid Mechanics 867, 906 (2019).

[18] F. Dabbagh, F. X. Trias, A. Gorobets, and A. Oliva, On the evolution of flow topology in turbulent Rayleigh-Bénard convection, Physics of Fluids 28, 115105 (2016).

[19] G. E. Elsinga and I. Marusic, Evolution and lifetimes of flow topology in a turbulent boundary layer, Physics of Fluids 22, 015102 (2010).

[20] H. M. Blackburn, N. N. Mansour, and B. J. Cantwell, Topology of fine-scale motions in 
turbulent channel flow, Journal of Fluid Mechanics 310, 269 (1996).

[21] J. Soria, R. Sondergaard, B. J. Cantwell, M. S. Chong, and A. E. Perry, A study of the fine-scale motions of incompressible time-developing mixing layers, Physics of Fluids 6, 871 (1994).

[22] C. B. da Silva and J. C. F. Pereira, Invariants of the velocity-gradient, rate-of-strain, and rate-of-rotation tensors across the turbulent/nonturbulent interface in jets, Physics of Fluids 20, 055101 (2008).

[23] F. X. Trias, F. Dabbagh, A. Gorobets, and C. D. Pérez-Segarra, Exploring new frontiers in Rayleigh-Bénard convection, (Ref. 2016163972), PRACE 15th Call (2017).

[24] A. Gorobets, F. X. Trias, R. Borrell, O. Lehmkuhl, and A. Oliva, Hybrid MPI+OpenMP parallelization of an FFT-based 3D Poisson solver with one periodic direction, Computers and Fluids 49, 101 (2011).

[25] R. W. C. P. Verstappen and A. E. P. Veldman, Symmetry-preserving discretization of turbulent flow, Journal of Computational Physics 187, 343 (2003).

[26] F. X. Trias and O. Lehmkuhl, A self-adaptive strategy for the time-integration of Navier-Stokes equations, Numerical Heat Transfer, part B 60, 116 (2011).

[27] R. J. A. M. Stevens, E. P. van der Poel, S. Grossmann, and D. Lohse, The unifying theory of scaling in thermal convection: the updated prefactors, Journal of Fluid Mechanics 730, 295 (2013).

[28] G. Grötzbach, Spatial resolution requirements for direct numerical simulation of the RayleighBénard convection, Journal of Computational Physics 49, 241 (1983).

[29] O. Shishkina, R. J. A. M. Stevens, S. Grossmann, and D. Lohse, Boundary layer structure in structure in turbulent thermal convection and consequences for the required numerical resolution, New Journal of Physics 12, 075022 (2010).

[30] B. Lüthi, M. Holzner, and A. Tsinober, Expanding the Q-R space to three dimensions, Journal of Fluid Mechanics 641, 497 (2009).

[31] F. X. Trias, D. Folch, A. Gorobets, and A. Oliva, Building proper invariants for eddy-viscosity subgrid-scale models, Physics of Fluids 27, 065103 (2015).

[32] P. Vieillefosse, Local interaction between vorticity and shear in a perfect incompressible fluid, Le Journal de Physique 43, 837 (1982).

[33] See supplemental material at, , for the conditional mean trajectories of small-scale dynamics 
in $\left(Q_{\mathrm{G}}, R_{\mathrm{S}}, \operatorname{tr}\left(\Omega^{2} \mathrm{~S}\right)\right)$ phase space. The trajectories are plotted for turbulent Rayleigh-Bénard convection at $R a=10^{10}$, in the bulk, and displayed in different facing views.

[34] A. Tsinober, M. Ortenberg, and L. Shtilman, On depression of nonlinearity in turbulence, Physics of Fluids 11, 2291 (1999).

[35] L. Chevillard, C. Meneveau, L. Biferale, and F. Toschi, Modeling the pressure Hessian and viscous Laplacian in turbulence: Comparisons with direct numerical simulation and implications on velocity gradient dynamics, Physics of Fluids 20, 101504 (2008).

[36] M. Wilczek and C. Meneveau, Pressure Hessian and viscous contributions to velocity gradient statistics based on Gaussian random fields, Journal of Fluid Mechanics 756, 191 (2014).

[37] Q. Zhou and K. Q. Xia, Physical and geometrical properties of thermal plumes in turbulent Rayleigh-Bénard convection, New Journal of Physics 12, 075006 (2010). 